\title{
Corela
}

Cognition, représentation, langage

HS-16 | 2015

Diversité des pratiques de recherche en science du langage

\section{Signe et signification à l'aune de la dichotomie syntaxe / sémantique}

\section{Manuel Gustavo Isaac}

\section{(2) OpenEdition}

Journals

Édition électronique

URL : http://journals.openedition.org/corela/3838

DOI : $10.4000 /$ corela.3838

ISSN : 1638-573X

Éditeur

Cercle linguistique du Centre et de l'Ouest - CerLICO

\section{Référence électronique}

Manuel Gustavo Isaac, "Signe et signification à l'aune de la dichotomie syntaxe / sémantique », Corela [En ligne], HS-16 | 2015, mis en ligne le 10 juillet 2015, consulté le 19 avril 2019. URL : http:// journals.openedition.org/corela/3838; DOI : 10.4000/corela.3838

Ce document a été généré automatiquement le 19 avril 2019

\section{(c) () (ㅇ)}

Corela - cognition, représentation, langage est mis à disposition selon les termes de la licence Creative Commons Attribution - Pas d'Utilisation Commerciale - Partage dans les Mêmes Conditions 4.0 International. 


\title{
Signe et signification à l'aune de la dichotomie syntaxe / sémantique
}

\author{
Manuel Gustavo Isaac
}

1 Dans le contexte de crise qu'est le tournant du $20^{\mathrm{e}}$ siècle, on assiste à l'émergence de trois projets visant à refonder l'approche théorique du langage en général. Tous trois sont basés sur une analyse du signe, comme objet théorique, et/ou de la signification, comme processus d'interprétation des signes et symboles des langages formels mathématiques et logiques. Tous trois ont leur ouvrage programmatique, établis selon des circonstances d'édition certes variables. Et enfin, tous trois partagent, de fait, des ambitions théoriques fondationnelles. Ces trois projets sont ceux de la sémiologie saussurienne, de l'idéographie frégéenne et de la grammaire pure logique de Husserl ${ }^{1}$. Leurs visées fondationnelles se situent à un niveau épistémologique. Pour autant, elles n'en ont pas moins une conséquence directe sur le mode d'appréhension théorique du signe comme objet. Cette conséquence, c'est la réduction du triangle sémiotique classique (langage, pensée, réalité) à des modélisations binaires du signe. Chacune des modélisations binaires en question est propre à chacun des trois projets. Et ce qui alors va se mettre en place, avec ces trois reconfigurations binaires de la structuration interne du signe, ce n'est rien moins que la dichotomie de la syntaxe et de la sémantique - cela, évidemment à chaque fois, selon des acceptions variables.

2 C'est la variation de ces acceptions en fonction du cadre théorique qu'elles intègrent (théorie du signe et/ou théorie de la signification) qui fait l'objet de cet article. Et puisque de fait, syntaxe et sémantique figurent désormais à l'interface de la logique et de la linguistique, on cherchera alors ici à établir la généalogie de leur divergence. Plus précisément, de la divergence entre deux conceptions de la notion de sémantique, une conception formelle ou formalisée d'inspiration logico-mathématique, et une conception morphologique et lexicale, affiliée quant à elle à la sémiologie structuraliste. 


\section{Le modèle sémiotique ternaire et la sémantique adéquationniste}

3 La modélisation du signe comme une structure ternaire constitue "l'horizon de rétrospection" des trois perspectives théoriques qui font l'objet de mon analyse. Ce modèle sert en fait ici de base à leur comparaison - et ce, précisément, en raison du fait que ces trois perspectives théoriques en opèrent la reconfiguration sur des modes binaires. Le modèle ternaire remonte à Aristote. On le trouve formulé dans les termes suivants aux premières lignes du traité De l'interprétation:

Les sons émis par la voix sont les symboles ( $\sigma u ́ \mu \beta o \lambda o v)$ des états de l'âme. Et de même que l'écriture n'est pas la même chez tous les hommes, les mots parlés ne sont pas non plus les mêmes, bien que les états de l'âme dont ces expressions sont les signes immédiats ( $\sigma \eta \mu \varepsilon i ̃ o v)$ soient identiques chez tous, comme sont identiques aussi les choses dont ces états sont les images. (Aristote, 2004 : 16a, 3-9)

La relation qui articule les trois sommets de ce "triangle sémiotique" est une relation dynamique, partant de la réalité pour atteindre le langage via la pensée. En tant que telle, cette relation est le processus dont résulte la signification des signes ou symboles du

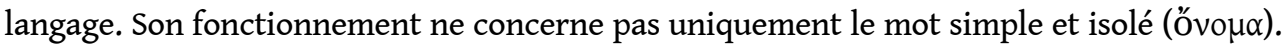
Il s'applique également au cas de la proposition ${ }^{2}$. Dans ce cas, il est alors possible d'établir un lien direct entre les signes ou symboles qui expriment la proposition et ce que la proposition signifie ou dénote, c'est-à-dire, la réalité. Et l'adéquation, ou la correspondance, des premiers avec la seconde est ce qui constitue pour Aristote le critère de validité sémantique de la proposition apophantique : «les propositions sont vraies en tant qu'elles se conforment aux choses mêmes (...).» (Aristote, 2004 : 18a, 33) ${ }^{3}$ Autrement dit, on a là, sur la base de la structuration ternaire du signe aristotélicien, une première version de la dualisation de la syntaxe et de la sémantique.

5 Cette dualisation forme un cadre de confrontation possible des théories de la signification de Husserl et Frege. C'est à partir des réductions qu'opèrent leurs modèles sémiotiques binaires respectifs par comparaison avec le modèle ternaire d'Aristote que l'on va pouvoir localiser leur opposition. Et c'est en opposant leur type de modèles sémiotiques binaires respectifs que l'on explique en quel sens et pourquoi leur paradigme théorique diffère radicalement de celui de l'analyse sémiologique de Saussure.

\section{Husserl et Frege : le paradigme de la logique formelle moderne}

6 Tel que je l'entends ici, le paradigme de la logique formelle moderne s'étend grosso modo des Lois de la pensée de Boole (1854) aux Principia mathematica de Russell \& Whitehead (1910). Il est ce cadre dans lequel se construit la formalisation mathématique de la logique via l'élaboration de systèmes symboliques concurrents. Husserl et Frege y ressortissent clairement (le second étant à l'origine d'innovations conceptuelles majeures, notamment quant à la théorisation de la quantification et à l'analyse du jugement). Cela, relativement à leur cadre de confrontation d'une part - en témoigne leur correspondance (Frege, 1978) -, et d'autre part, en raison de leurs filiations en termes de théorie de la signification. Sur ce point, leurs perspectives sont à terme frontalement opposées. En effet, l'extensionnalisation progressive de la logique de Frege à partir de 1884 corrélée à 
l'affirmation de la prévalence de la sémantique sur la syntaxe dans les années 1892 préfigure en quelque sorte la sémantique modèle-théorique ${ }^{4}$, tandis que l'intensionnalisation progressive du calcul des significations de Husserl corrélée à la "monicisation" de la syntaxe et de la sémantique constitue le socle du développement de sémantiques de type preuve-théorique ${ }^{5}$. La première étant mieux connue, on traitera en général par la suite plus longuement du cas de Husserl.

\subsection{Deux modèles binaires du signe}

7 Afin de mettre les choses au point dès le départ, on va commencer par traiter ce qui est au principe des projets de Husserl et Frege, à savoir, dans chacun des cas, un ensemble de considérations philosophiques et épistémologiques sur le statut et la fonction de la logique. Ces considérations englobent autant une définition de l'objet logique formel en tant que tel que, par ailleurs, dans le cadre d'une théorie de la science, la caractérisation de son rôle d'instrument. Et c'est de cette dernière que s'ensuivra le développement des conceptions binaires du signe propres à chacun des deux auteurs. Dans chacun des cas, celui de Husserl aussi bien que celui de Frege, on a affaire à un programme de type fondationnaliste. C'est à chaque fois par cet angle qu'on les aborde.

\subsubsection{Le fondationnalisme}

Dans le cas de Frege, on se focalise sur la première idéographie (Frege, 1879). Le projet y est de fonder l'arithmétique sur la logique. C'est à ce titre qu'on parlera de "logicisme". Et c'est au titre du projet logiciste que, dans ce cadre, les constructions déductives des propositions de l'arithmétique sont réduites à la dérivation logique. Dès lors, ce qu'il va falloir, c'est se doter d'un instrument d'analyse précis des chaînes d'inférences afin d'en révéler les implicites. L'idéographie est cet instrument. Car en tant que "langage formulaire de la pensée pure" ayant, qui plus est, « renonc[é] à exprimer tout ce qui est sans signification pour la déduction» (Frege, 1999: 6), elle est censée attester de la formation d'un mode de désignation adapté aux choses mêmes de l'arithmétique - et plus généralement de la raison: car avec l'arithmétique, la raison n'a affaire qu'avec ellemême, ou encore, est à elle-même son propre objet (Frege, 1879 : \$105). Et précisément, la finalité épistémologique de l'idéographie est là; elle consiste dans la réduction significative de l'écart entre pensée pure et expression formelle. Tel est le statut de l'idéographie comme instrument au service de la réalisation du projet logiciste, autrement dit, en tant qu'il lui est coordonné une théorie de la science. Cette théorie est exemplairement développée par Frege (1882a), dont la thèse est aussi explicite que le titre: les sciences abstraites ont besoin de se doter d'un système sémiotique efficace, stable et précis, afin d'assurer la désambiguïsation de leurs dérivations. Clairement, il s'ensuit de ces constatations que le programme logiciste de 1879 opère un "tournant symboliste". La stratégie argumentative générale de Frege (1884) corrobore cette perspective: l'hypothèse est que l'arithmétique est la science de la pensée pure; le corollaire, que le réductionnisme logique est possible; et la conclusion, que l'analyse du fonctionnement symbolique $\mathrm{du}$ concept de nombre explicite le mode général de formation des concepts. On est bien en train de fonder, par la logique, les mathématiques, et via les mathématiques, la science elle-même. Et la question sémiotique est au centre d'un tel programme. 
9 Sur le principe, le problème de Husserl à ses débuts est similaire, et son objet est le même : il s'agit de fonder l'arithmétique (Husserl, 1891a). Cela passe ici aussi par l'analyse des concepts fondamentaux de cette science - le but étant à terme d'en clore le système (Husserl, 1887). Et à nouveau, le symbolisme en constitue l'enjeu central. À cela, deux raisons : premièrement, en tant qu'il forme le double principe articulant la science arithmétique, à savoir sa condition de conceptualisation et de représentation; deuxièmement et surtout, en tant qu'il formalise la transition du concept au jugement, c'est-à-dire, par analogie, le passage du signe à la formule (Husserl, 1887). Ainsi, c'est parce que les processus cognitifs reposent sur de telles bases signitives (sic) pures qu'élaborer une "théorie universelle du sens » suppose de déterminer les conditions de possibilité de la pensée symbolique, et en ce sens, la délimitation de la sphère de la logique pure répond à des fins d'ordre transcendantal (Husserl, 1939). Ce sont les Recherches logiques (1901) qui, à ce niveau, prolongent le programme fondationnaliste des années 1890 en élargissant sa portée à l'enquête de l'essence rationnelle de toute science déductive - l'objectif étant d'élaborer une théorie générale des systèmes déductifs formels, et par là, de formuler l'essence des lois formelles de la connaissance (Husserl, 1901a : Vorwort). Cet objectif est au principe du projet d'élaborer une « logique pure » qui, en tant que système théorique unitaire et unifié, doit se constituer comme totalité idéalement fermée de lois ${ }^{6}$. Ce qui est alors en germe avec la délimitation de cette sphère logico-idéale pure - «sphère idéale d'être et de science » déterminant l'idéalité objectale des objectités existantes -, c'est l'idée de l'existence d'une corrélation apriorique formelle et d'ordre nomologique entre catégories de significations et catégories d'objets. Ainsi, de la logique à l'ontologie, ce qui se met en place, c'est une théorie transcendantale des catégories du sens et de la signification. Mais avant d'en arriver là, analysons dès maintenant l'impact d'un tel projet de fondation sur la redéfinition de la structuration interne du signe ${ }^{7}$.

\subsubsection{Le "réductionnisme"}

Dans le cas du premier Husserl, celui des années 1890, la réduction de la structure ternaire du signe à un modèle binaire tient au statut secondaire de la question du référent. La secondarité est ici à entendre au sens propre: elle est ce qu'on dérive d'un premier. Et le premier, c'est évidemment le sens qui intervient dès Husserl (1891a), dans le cadre du traitement des niveaux esthétiques inférieurs du fonctionnement de l'intentionnalité (temps et espace). Dans ce cadre, le point de départ de la signifiance se situe au niveau des phénomènes, phénomènes qui, en tant que donnés sous un aspect déterminé, sont non seulement principes mais également moyens de la signification. Et c'est précisément en tant que tels que pour être signifiant(s) - c'est-à-dire, pour fonctionner comme indices sémantiques -, ils supposent une modification intentionnelle opérée par le sujet de la connaissance : la Bedeutung (sens ou signification). Cette dernière fonctionne alors comme le facteur du surgissement d'un "noyau de sens idéal" (miperception, mi-signification), et apparaît en conséquence comme un principe de déséquilibre d'importance introduit dans le champ objectif par l'activité conscientielle de thématisation intentionnelle (anté- ou proto-sémiotique) du sujet de la connaissance ${ }^{8}$. Tout cela mène (Husserl, $1891: 2$. Theil) à une théorie de la représentation dans laquelle la représentation, comme facteur d'idéalisation, est fondamentalement impropre, car toujours virtuellement symbolisante. Dès lors, si selon Husserl construire un signe consiste à fixer des marques (Husserl, 1970b) - ce marquage distinctif est même ce qui 
détermine la valeur sémiotique -, c'est donc par remplacement de la donation effective et directe de l'objet intuitif que la représentation opère. Comme telle, la représentation fonctionne en tant qu'opérateur de description posant une relation d'équivalence entre le propre et le symbolique modulo un principe de substitution (Husserl, 1894). On retrouve une telle conception substitutiviste du signe en Husserl (1901b : \$54) : étranger à la chose par son contenu, le signe y est défini par sa fonction d'évocation du désigné, capable de doter de présence fictive le symbolisé, alors remplacé par représentation. C'est donc bien à une supplantation du référent comme pôle sémiotique que procède Husserl en articulant alors simplement la binarité du langage et de la pensée?

Quant à Frege, son projet de refondation du formalisme de la logique repose sur une double exclusion de principe, celle du psychologique et de la subjectivité (Frege, 1884 : Einleitung). Aussi, la réduction du modèle sémiotique ternaire va consister dans son cas à évacuer hors du calcul du sens le pôle de la pensée, dans sa dimension subjective ${ }^{10}$. Autrement dit, à exclure les représentations. C'est que, selon lui, si l'on ne distingue pas entre choses et représentations des choses (dont elles sont le corrélat subjectif de la saisie), tout se confond. Les premières sont traitées comme objectivités, tandis que la réalité objective même est elle-même traitée comme représentation ${ }^{11}$. Dès lors, la reconfiguration de la structure sémiotique mise en place par l'Idéographie (1879) va s'opérer par l'assimilation de la dimension objective et conceptuelle de la pensée avec la dénotation référentielle pour constituer le plan de la sémantique, plan quant à lui distinct de l'écriture qui l'exprime comme son contenu (la Begriffsschrift). On a donc, d'un côté le pur symbolisme des signes du langage, de l'autre ce que ces signes signifient indistinctement, concept et réalité. En d'autres termes: une première version de la dichotomie de la syntaxe et de la sémantique.

\subsection{La dichotomie syntaxe / sémantique : intension versus extension}

Dans le prolongement des sous-sections précédentes, on trace ici la dichotomie inchoative de la syntaxe et de la sémantique, respectivement chez Husserl (1891-1901) et Frege (1879-1892). Afin d'en faciliter l'appréhension, signalons que le mouvement des deux auteurs est asymétrique, en chiasme : tandis qu'avec Husserl, on part d'une dualité nette pour aboutir à un "monisme" fusionnel - celui de l'inten[s/t]ionnalité -, Frege cale progressivement sa "dualité moniste" de départ sur une dichotomie stricte.

\subsubsection{Husserl (1890-1900/1) : De la dualité au monisme (internaliste)}

13 Avec Husserl, on a clairement affaire à une conception substitutiviste du fonctionnement des systèmes symboliques - exemplairement en Husserl (1970b). Et pour cette raison, le problème de la mise en place de l'interaction de la syntaxe et de la sémantique y est évidemment central, car ce qu'il faut établir, c'est une correspondance stricte (ou isomorphisme) entre le substitut et ce à quoi il se substitue afin de garantir la régularité de leurs équivalences, en l'occurrence, calculatoires. Cela peut s'envisager de deux manières: soit on part de deux systèmes considérés comme hétérogènes, et on se concentre sur le développement d'un processus de traduction-interprétation qui les articule ; soit on considère qu'un seul et même système est susceptible des deux types de fonctionnement (syntaxique et sémantique), et ce relativement à la perspective selon laquelle on l'appréhende. La transition de la première à la seconde manière d'envisager la 
mise en relation des deux systèmes n'est rien d'autre que la transition d'une conception dichotomique et dualisée de l'articulation de la syntaxe et de la sémantique à leur fusion moniste. Autrement dit: la progression de la Philosophie de l'arithmétique (1891) aux Recherches logiques (1901).

Dans la Philosophie de l'arithmétique (Husserl, 1891a: chap.12), le parallélisme entre sémiotisation (système de désignations) et sémantisation (système de concepts) s'opère par adéquation-conformation de la première à la seconde - autrement dit, le mode de formation des concepts prime sur le mode de désignation (voir infra). Pour autant, dans ce même cadre, la construction récursive (c'est-à-dire par induction sur la complexité des compositions générées) de la correspondance des deux systèmes est gérée au niveau des règles propres à chacun des systèmes. Or, ce que cette autonomie réciproque des deux systèmes implique, c'est l'indépendance formelle de leurs opérations mécaniques respectives. En sorte qu'à partir de là, on obtient la possibilité d'un développement indépendant du système syntaxique ${ }^{12}$ (modulo la fixation par convention d'équivalences sémiotiques de départ). Par cette autonomisation, la syntaxe se constitue comme un processus mécanique externalisé (re)produisant la formation mentale des concepts, mais indépendamment, en termes calculatoires, des concepts de l'esprit: on substitue au processus sémantique de production de concepts le processus de déduction systématique des symboles, et ce n'est qu'au terme de la dérivation que la correspondance se rétablit par transformation inverse (des symboles dans les concepts). Ainsi, du point de vue de l'articulation de la syntaxe et de la sémantique, Husserl (1891a) est radicalement dichotomique. Le calcul logique y est défini comme un ensemble de signes arbitraires et sans signification assurant la construction uniforme d'un système de déduction total et clos, sémantisable a volo. Dans le même sens, pour Husserl (1891b), calculer procède par substitution symbolique aveugle et règles mécaniques. Cependant, Husserl insiste dans ce cas sur le fait que le fondement de la conséquence logique réside dans son interprétation, et donc, que la formalisation algorithmique des déductions n'a de valeur que par sa convertibilité sémantique. C'est que le calcul pur de la dérivation ne représente pas la totalité du processus de déduction, mais qu'il suppose d'avoir d'abord procédé à la "mise en équation" du système de calcul avec (les objets de) l'activité véritable du jugement, à savoir les concepts. Et puisque ces concepts sont alors supposés être de nature extensionnelle, constituer la déduction sous forme calculatoire passe par l'extensionnalisation du jugement de contenu en jugement de classes.

Deux inédits de la même période (Husserl, 1970c; Husserl, 1970d) développent ce parallélisme des conceptions intensionnelle et extensionnelle de la signification en théorisant un ensemble de procédures de substitution sémiotique. Le processus de déduction y est dit comporter trois phases : la mise en équation intension / extension; le calcul à proprement parler (la dérivation); l'interprétation ou sémantisation du résultat. Et sur la base de cet ensemble d'équivalences sémiotiques par substitution va véritablement se développer l'indépendance calculatoire de la syntaxe. C'est le cas de manière exemplaire en Husserl (1891 : chap. 13) où cette mise en équation de l'intension et de l'extension est analysée comme la mise en correspondance de deux méthodes de déduction, respectivement syntaxique et sémantique. Leur articulation s'opère alors sur le mode d'une double dynamique, onomasiologique d'abord, puis sémasiologique; soit, par «conversion des pensées de départ en signes - calcul - conversions de signes résultant en pensée. » (Husserl, 1992: 319). Et si cela concrétise l'indépendance de la syntaxe comme productrice de sens, c'est que le mécanisme calculatoire, procédant par 
substitution systématique de signes aux concepts et opérant par là immédiatement sur une extériorité formelle, génère des conséquences sémantiques rétroactivement, c'est-àdire au moyen d'une (ré)interprétation des équivalences fixées au départ ${ }^{13}$. Il y a donc bien, ici aussi, conversion-déduction-reconversion. Mais avec cette fois, prévalence du calcul sur la sémantique. À partir de là, Husserl va projeter l'unification des deux types de systèmes déductifs, sémantique et syntaxique, en un "calcul logique véritable ». Elle s'opérera en rétrocédant en deçà de l'alternative entre intension et extension pour finalement atteindre leur base calculatoire commune : « le calcul (pur) des Multiplicités » (Mengencalcul). Une telle "identité algorithmique» (Husserl, 1995: 86) signifie que désormais, appliqué indifféremment aux classes et aux contenus de concepts, «le même calcul reçoit [simplement] un autre sens dans la résolution de mêmes problèmes." (Husserl, 1995 : 68-69) Cette unification calculatoire est ce sur quoi est scellée en Husserl (1891c) la fusion entre syntaxe et sémantique : la syntaxe du calcul logique est non pas interprétée mais signifie directement les conditions de la validité formelle des jugements.

En somme, les inédits de la période 1890-1900 s'organisent toujours autour de la même tension. Soit: pour le système sémiotique, être processus mécanique (algorithme) producteur de "sens" versus pour le système sémantique, être le principe d'institution du "sens". La période des Recherches logiques figure d'une certaine manière la résolution de cette tension. Et cela, en densifiant la fusion de la syntaxe et de la sémantique. De manière exemplaire en Husserl (1970e), le calcul logique ne va plus consister dans le traitement de propositions considérées comme sémantiquement neutres, c'est-à-dire antérieurement à leur évaluation vériconditionnelle. Non, désormais, c'est sur les valeurs de vérité, internalisées par les propositions, que l'on va alors opérer lorsque l'on compose les prédications (niveau de la morphologie formulaire), ou encore, de façon plus évidente, que l'on va calculer indirectement lorsque dans la dérivation de preuves, on déduit telle ou telle proposition (niveau de la syntaxe des preuves). Dès lors, la syntaxe n'est plus subordonnée au vériconditionnel d'ordre sémantique, mais bien au contraire, elle se l'est assimilée. La syntaxe apparaît alors comme le principe opératoire et producteur de la vériconditionalité. Et c'est en tant que forme pure conditionnant les lois des valeurs de vérité que son nom s'élargit pour devenir « Grammaire Pure Logique » (Husserl, 1901). La focale s'est ainsi déplacée d'une polarisation sur la dualité de la syntaxe et de sémantique à leur "monicisation" grammaticale pure, pour finalement articuler ce calcul pur des significations, via l'élaboration proprement phénoménologique de la notion d'intentionnalité, avec le référent (réel ou non) qu'il est susceptible de viser.

\subsubsection{Frege (1879-1892) : Du monisme (dualiste) à la dichotomie}

Parler de monisme dualiste, dans le cas de Frege (1879), se réfère au fait que, lorsqu'il cadre son projet en préface, il assigne à son langage formulaire la double tâche d'être à la fois caractéristique universelle (lingua characterica) et calcul (calculus philosophicus / ratiocinator). En 1879, calcul et caractéristique sont indistincts, de même que philosophicus et ratiocinator sont identifiés. Cela signifie qu'à ce moment, le formalisme idéographique est tout autant constitué de règles logiques d'usage calculatoire qu'il doit par ailleurs être apte à exprimer les contenus conceptuels et de jugements (begriffliche und beurteilbare Inhalte). En cherchant à clarifier l'articulation de ces deux dimensions, on conçoit que l'idéographie comme caractéristique est l'opérateur de dépiction et d'expression logiques des contenus de pensée (ou concepts), tandis que ses symboles propres sont les signes du calcul propositionnel des contenus de jugement. C'est là selon Frege (post-1879) la 
puissance même de l'idéographie: non seulement son symbolisme est au principe de l'analyse de la pensée, mais encore, il permet la représentation simultanée de l'intégralité des données (structurelles) du raisonnement. En d'autres termes, on peut penser directement dans les symboles - eux-mêmes représentants de la pensée. D'où il s'ensuit à l'évidence que l'on assiste ici également à la mise en place de l'interaction de la syntaxe et la sémantique. Mais ce qui précisément change dans ce cadre, c'est que leur interaction est unifiée à même le symbolisme de l'idéographie.

En 1879, c'est la gestion du rapport entre règles logiques et axiomes qui permet le mieux de visualiser l'unification de l'interaction de la syntaxe et de la sémantique au sein du système formel de l'idéographie. Pour Frege, la fixation des règles du calcul est immotivée si elle n'est pas fondée sémantiquement. Et comme les signes d'une caractéristique ne sont, par principe, ni vides ni arbitraires, la base unique de toute déduction doit être le symbolisme, moyennant les règles. C'est pourquoi, pour conjuguer en l'idéographie fonctionnement calculatoire et statut de caractéristique, Frege veut un minimum de règles (en 1879, uniquement le modus ponens), et aura par conséquent un maximum d'axiomes - les axiomes fonctionnant comme facteurs de motivation sémantique du calcul en tant qu'ils représentent les lois de la pensée. On voit clairement qu'ici se joue l'insertion de la sémantique dans la syntaxe, ou encore la sémantisation de la syntaxe, mais sur un plan d'abord interne et immanent au symbolisme même. Et c'est au niveau même du symbolisme que cette articulation interne de la syntaxe et de la sémantique est scellée par le traitement de la bidimensionnalité graphique. Cela, de telle sorte que, si Frege (1882b), en réponse à Schröder, clarifie enfin le clivage de la caractéristique et du calcul - la première étant conçue comme une traduction réfléchissant les propriétés des objets symbolisés ; le second, identifié à un artificium facile et infaillibilter ratiocinandi -, ce n'est que pour mieux signaler leur indissociabilité. Et de cette indissociabilité, justement, les deux dimensions de la page en sont le plan d'articulation: la verticalité, symbolisant les formes logiques abstraites, exprime les rapports morpho-syntaxiques; tandis que l'horizontalité, lieu de la substitution, exprime le contenu sémantique.

En résumé et à l'instar de Husserl, il y a en permanence cette tension interne au réductionnisme de Frege : d'une part, « calculer serait déduire » (Frege, 1969 : 211) tandis qu'en parallèle on est supposé pouvoir «substituer les idées au calcul » ainsi que l'air du temps l'impose. Durant cette période, Frege balance sans se décider entre le fait d'identifier la logique formelle abstraite aux rapports propositionnels et la simple subordination desdits rapports aux contenus propositionnels (valeurs de vérité). Autrement dit, Frege ne choisit pas entre reconnaître une syntaxe à la signification endogène, ou alors au contraire, affirmer la prérogative de la sémantique sur la syntaxe. Car ce que l'on veut, c'est limiter l'arbitraire attribué aux règles du calcul par leur motivation sémantique et définitionnelle pour en ériger les formes fixes en lois logiques, par assimilation donc. Mais on reste incapable d'assigner la différence matérielle entre calcul symbolique pur et preuve logique formelle sémantiquement valide. L'extensionnalisation progressive de la logique de Frege va clarifier ce point en causant directement la dichotomisation de la syntaxe et de la sémantique ${ }^{14}$ pour aboutir à leur quasi dualisation sous la forme du diptyque sens / dénotation (Frege, 1892a), avec au final, subordination de la syntaxe à la sémantique. Ainsi, de 1879 au années 1890, le développement de la philosophie de la logique de Frege forme bien un chiasme avec celui de Husserl. Ce chiasme est localisable sur des points théoriques précis, et comme tel, 
inscrit leur confrontation au registre d'un même paradigme - celui de la logique formelle moderne. C'est ce paradigme que l'on va maintenant comparer à celui de Saussure.

\section{Saussure : la paradigme de la sémiologie}

Mes analyses se concentrent ici sur les extraits de textes où la linguistique intègre clairement le projet d'une sémiologie entendue comme science des signes, c'est-à-dire où la linguistique est clairement identifiée à la théorie d'un système de signes (Saussure, $1968: 169 f^{15} / 2002: 220$ ). Je le fais ici en me focalisant sur l'organisation systémique des extraits de textes en question - organisation faite de principes, de conséquences, de corollaires - afin d'en envisager la comparaison avec les projets plus ou moins formalisés de Husserl et Frege, du moins en ce qui concerne la mise en place de la dichotomie syntaxe / sémantique.

\subsection{Le fondationnalisme saussurien : "axiomatisation" et perspectivisme}

Lorsque Saussure formule son projet d'une théorie générale du langage au cours des années 1890 - cf. la lettre à Meillet, approximativement datable de 1894 (Godel, 1957 : 31) -, il lui impose alors deux préalables : d'une part, la classification "logique" des faits de langage, de l'autre, la catégorisation des opérations du linguiste. Dans les deux cas, l'objectif de Saussure relève du fondationnalisme. C'est explicite d'abord au niveau de l'articulation générale de la théorie, en raison de l'idéal saussurien d'une linguistique construite comme "pure science de rapports abstraits» (Saussure, 1968: 173f / 2002 : 334) dans laquelle tout serait système ${ }^{16}$, c'est-à-dire où l'on partirait d'axiomes pour dériver des théorèmes, obtenir des corollaires (Godel, 1957: 3017), et au final, pouvoir prédire en théorie l'intégralité des déplacements sémiologiques possibles (Saussure, 1974 : 22b / $2002: 206$ ). C'est explicite, ensuite, au niveau de la détermination de l'objet de la théorie (Saussure, 1980:16) en raison de la position perspectiviste que le maitre de Genève adopte. Cette position consiste à " poser que rien n'est donné » (Godel, $1957: 42$ ), en l'occurrence, qu'aucun objet dans la langue n'est un donné antérieur à l'analyse et indifférent à sa théorisation ${ }^{18}$. Dans ce cadre, le seul point de départ pour la science du langage, c'est la théorie elle-même, car "Bien loin que l'objet précède le point de vue, c'est le point de vue qui crée l'objet. » (Saussure, $1980: 23$ ) Ce qui veut dire qu'on ne se rapporte au langage, dans la pratique de sa théorisation, que par le processus de sémiotisation opéré sur lui par la conscience qui le thématise : il faut exister et être perçu comme signe par la conscience pour participer de l'ordre linguistique ${ }^{19}$ (Saussure, 2011: 103 / 2002: 45) - ou encore, pouvoir-être implique ici devoir-avoir un sens. Et c'est justement à propos de l'indexation sémantique du signe linguistique qu'intervient l'exclusion du référent réel hors du domaine de la langue. En gros, l'antiréalisme saussurien est transféré du cadre épistémologique à celui de l'ontologie via la construction théorique de la sémiologie linguistique.

\subsection{La réduction du modèle ternaire : exclure le référent}

Si, appliqué à la langue - considérée comme cas particulier, certes « le plus complexe, le plus répandu [...], le plus caractéristique [...] (Saussure, $1980: 101)$, de l'ensemble des 
systèmes de signes -, le projet sémiologique se fonde sur la condamnation du traitement théorique du référent, c'est parce que pour Saussure le modèle de la signification de la langue n'est pas celui de la nomenclature: «le fond du langage n'est pas constitué de noms. [Et] c'est un accident quand le signe linguistique se trouve correspondre à un objet défini pour les sens [...] plutôt qu'à une idée.» (Saussure, $1968:$ 148f / $2002: 230$ ) Un tel rejet est cohérent avec son épistémologie, car, de fait, théoriser la langue comme nomenclature consiste à en indexer la sémantique sur le réel comme base extérieure donnée ${ }^{20}$ - ce qui, évidemment, contredit l'idée selon laquelle les objets de la linguistique n'existent que par leur mise en perspective théorique. C'est en ce sens que l'antiobjectivisme saussurien est transféré de la sphère de l'épistémologie de la linguistique, dans laquelle il engage l'idéalisation de son matériau, à la sphère ontologique, dans laquelle il déclenche l'oblitération de la question du référent, via la construction théorique de la sémiologie. La question du référent est donc exclue du cadre d'une linguistique conçue comme théorie d'un système de signes ${ }^{21}$. Dans ce champ, il n'y a donc pas de bijection entre les éléments du langage et ceux de la réalité, pas d'isomorphisme, donc, entre leurs structures respectives ${ }^{22}$. En somme, le signe linguistique se réduit à l'articulation d'une binarité, celle du signifiant et du signifié. Et c'est au niveau de son insertion dans le système de la langue que va se déterminer la signification des unités sémiotiques bifides.

\subsection{La dichotomie syntaxe / sémantique et les deux principes de la sémiologie}

Que la sémantique saussurienne s'élabore au niveau du système-langue, ce n'est là que le contrecoup de l'absence de toute positivité sémantique, référentielle aussi bien que conceptuelle ${ }^{23}$. Dès lors, seul le jeu interne d'un système basé sur des rapports oppositifs, négatifs, différentiels - soit sur le quaternion (Saussure 2011: 150, 154 / Saussure, 2002 : $73,39)$ institué en schème de la structure sémiologique de la langue - peut être au principe de la détermination de la valeur des unités sémiotiques. Ce système est régi par deux principes, l'arbitraire et la linéarité. Et ces deux principes sont eux-mêmes hiérarchisés. C'est à leur niveau que va s'appliquer la dichotomie de la syntaxe et de la sémantique. Et c'est relativement à leur hiérarchie que va se déterminer le type de leur articulation. Cette dernière fonctionne autant du point de vue sémasiologique qu'onomasiologique. Dans les deux sens, elle a une explication triviale en termes ensemblistes. Soit: sur la base d'une dualité de masses amorphes (pensée et matière phonique) ordonnée par une relation préordre (réflexivité, transitivité), on restreint cette dernière à ses points de symétrie pour obtenir une relation d'équivalence $\mathbf{R}$ (c'est-à-dire une relation binaire concept / image acoustique ${ }^{24}$ ); modulo $\mathbf{R}$, on passe au quotient l'ensemble de départ pour en obtenir la partition en classes d'équivalence (Sa/Sé) : finalement, parce que celles-ci sont articulées par un ordre large (réflexif, transitif, antisymétrique), on en dérive un ordre strict (irréflexif, transitif, asymétrique) pour obtenir la linéarité. L'inverse n'est pas possible. Ce que cette explication manifeste, c'est que l'application du principe de linéarité, en tant qu'il est secondaire, engage une dynamique orthogonale entre l'horizontalité de la ligne ordonnée et la verticalité de l'association interne du signe. Cette dynamique orthogonale ne figure rien d'autre que l'interaction internalisée des deux principes de la sémiologie au niveau du système autrement dit: de la syntaxe et de la sémantique ${ }^{25}$. C'est cette internalisation systémique 
de la syntaxe et de la sémantique, et la dynamique de l'orthogonalité des deux principes sur laquelle elle repose, qui distingue le paradigme sémiologique de l'analyse saussurienne des modélisations dichotomiques et dualistes de Frege et Husserl.

\section{Conclusion}

Ma conclusion tient en deux observations. La première concerne ce que j'ai qualifié de "paradigme de la logique formelle moderne". Elle résulte de l'analyse du chiasme structurant la confrontation Husserl-Frege, et consiste dans le simple constat suivant: plus on extensionnalise un formalisme logique (et la théorie de la signification qui lui est reliée), plus on dichotomise la relation de la syntaxe et de la sémantique, tandis que plus on intensionnalise un tel formalisme (et la théorie de la signification qui lui est reliée), plus on opère la fusion moniste de la syntaxe et de la sémantique. Quant à la seconde observation, elle consiste à affirmer que la prise en compte de la conséquence majeure de la dynamique de ce chiasme (intension versus extension) - à savoir, la mise en place de l'objectif de la dualisation de la syntaxe et de la sémantique (en fonction de perspectives évidemment opposées) - par la sémiologie est en quelque sorte obérée par le fait que sa propre conception du signe implique l'internalisation de la relation de la syntaxe et de la sémantique au niveau des principes du système que le signe intègre en tant qu'élément. Et cela, de telle sorte qu'on aurait ici une explication possible de la divergence, dès avant le tournant $\mathrm{du} 20^{\mathrm{e}}$ siècle, entre deux conceptions de la sémantique, l'une formelle et mathématique, l'autre structurale ou structuraliste ${ }^{26}$.

\section{BIBLIOGRAPHIE}

AJDUKIEWICZ, Kazimierz (1935), « Die syntaktische Konnexität », Studia Philosophica, 1, 1-27.

ARISTOTE (2004), De l'interprétation, trad. J. Tricot, Paris : Vrin.

ARISTOTE (1991), Métaphysique, trad. J. Tricot, Paris : Vrin.

BAR-HILLEL, Yehoshua (1953), «A quasi-arithmetical notation for syntactic description », Language, $29,47-58$

BOOLE, George (1854), An investigation of the laws of thought: on which are founded the mathematical theories of logic and probabilities, London: Walton and Maberly; Cambridge: MacMillan.

CURIEN, Pierre-Louis (2004), « Introduction to linear logic and ludics » [en ligne], 2 parts, in Programmes, Preuves et Systèmes (UMR-CNRS 7126) - Université Paris 7 (page personnelle) [consulté le 15 mars 2012], http://www.pps.jussieu.fr/ curien/LL-ludintroI.pdf et http://www.pps.jussieu.fr/ $\sim$ curien/LL-ludintroII.pdf.

ENGLER, Rudolf (1968), Lexique de terminologie saussurienne, Utrecht / Anvers : Het Spectrum.

FREGE, Gottlob (1879), Begriffsschrift, eine der arithmetischen nachgebildete Formelsprache des reinen Denkens, Halle a/S. : L. Nebert. 
FREGE, Gottlob (1882a), « Über die wissenschaftliche Berechtigung einer Begriffsschrift », Zeitschrift für Philosophie und philosophische Kritik, 81, 48-56.

FREGE, Gottlob (1882b), « Über den Zweck der Begriffsschrift », Vortrag, gehalten in der Sitzung vom 27. Januar 1882 der Jenaischen Gesellschaft für Medizin und Naturwissenschaft, Jenaische Zeitschrift für Naturwissenschaft, 16/Supplement, 1-10.

FREGE, Gottlob (1884), Die Grundlagen der Arithmetik: eine logisch mathematische Untersuchung über den Begriff der Zahl, Breslau : W. Koebner.

FREGE, Gottlob (1891), Funktion und Begriff, Vortrag, gehalten in der Sitzung vom 9. Januar 1891 der Jenaischen Gesellschaft für Medizin und Naturwissenschaft, Jena : Hermann Pohle.

FREGE, Gottlob (1892a), « Über Sinn und Bedeutung », Zeitschrift für Philosophie und philosophische Kritik, 100, 25-50.

FREGE, Gottlob (1892b), « Über Begriff und Gegenstand », Vierteljahresschrift für wissenschaftliche Philosophie, 16, 192-205.

FREGE, Gottlob (1893), Grundgesetze der Arithmetik. Begriffsschriftlich abgeleitet, I. Band, Jena : H. Pohle.

FREGE, Gottlob (1894), « Rezension von : E. Husserl, Philosophie der Arithmetik I », Zeitschrift für Philosophie und philosophische Kritik, 103, 313-332.

FREGE, Gottlob (1903), Grundgesetze der Arithmetik. Begriffsschriftlich abgeleitet, II. Band, Jena : H. Pohle.

FREGE, Gottlob (1969), Les fondements de l'arithmétique : recherche logico-mathématique sur le concept de nombre, trad. C. Imbert, Paris : Seuil.

FREGE, Gottlob (1987), Correspondance : Gottlob Frege-Edmund Husserl, trad. G. Granel, Mauvezin : TER.

FREGE, Gottlob (1999), Idéographie, trad. C.Besson, Paris : Vrin.

GIRARD, Jean-Yves (2001), « Locus solum », Mathematical Structures in Computer Science, 11, 301-506

GIRARD, Jean-Yves (2007), Le point aveugle. Vers l'imperfection, t. 2, Paris : Hermann.

VAN HEIJENOORT, Jean (1967), « Logic as calculus, logic as language », Synthese, 17/1, 324-330.

HUSSERL, Edmund (1887), Über den Begriff der Zahl : psychologische Analysen, Habilitationsschrift durch welche... zu seiner... Antrittsvorlesung über die Ziele und Aufgaben der Metaphysik... einladet Dr. Edmund G. Husserl, Halle a./S : F. Beyer.

HUSSERL, Edmund (1891a), Philosophie der Arithmetik, psychologische und logische Untersuchungen, Halle a./S : R. Stricker.

HUSSERL, Edmund (1891b), « Besprechung. Schröder, Ernst : Vorlesungen über die Algebra der Logik », Göttingische gelehrte Anzeigen, 7, 243-258.

HUSSERL, Edmund (1891c), « Der Folgerungscalcul und die Inhaltslogik », Vierteljahrschrift für wissenschafliche Philosophie, 15, 168-189.

HUSSERL, Edmund (1893), « A. Voigts 'elementare Logik' und meine Darlegungen zur Logik des logischen Calcul », Vierteljahrschrift für wissenschafliche Philosophie, 17, 111-120.

HUSSERL, Edmund (1894), « Psychologische Studien zur elementaren Logik », Philosophische

Monatschefte, 30, 159-191.

Corela, HS-16 | 2015 
HUSSERL, Edmund (1900), « Husserl, E. G. : Logische Untersuchungen. Erster Teil : Prolegomena zur Reinen Logik », Selbstanzeigen, Vierteljahrschrift für wissenschafliche Philosophie, 15, 511-512.

HUSSERL, Edmund (1901a), Logische Untersuchungen. Erster Band. Prolegomena zur reinen Logik, Halle a./S : M. Niemeyer.

HUSSERL, Edmund (1901b), Logische Untersuchungen. Zweiter Band. Untersuchungen zur Phänomenologie und Theorie der Erkenntnis. V. Über intentionale Erlebnisse und ihre „Inhalte“, Halle a./ S : M. Niemeyer.

HUSSERL, Edmund (1901c), Logische Untersuchungen. Zweiter Band. Untersuchungen zur Phänomenologie und Theorie der Erkenntnis. V. Über intentionale Erlebnisse und ihre „Inhalte“, Halle a./S : M. Niemeyer. HUSSERL, Edmund (1901d), « Husserl, E. G. : Logische Untersuchungen. Zweiter Teil : Untersuchungen zur Phänomenologie und Theorie der Erkenntnis », Selbstanzeigen, Vierteljahrschrift für wissenschafliche Philosophie, 15, 260-263.

HUSSERL, Edmund (1939), « Esquisse d'une préface aux Recherches logiques (1913) », Tijdschrift voor Philosophie, I, 106-133, 319-339.

HUSSERL, Edmund (1970a), Philosophie der Arithmetik : mit ergänzenden Texten, 1890-1901

[Husserliana : 12], Den Haag : Martinus Nijhoff.

HUSSERL, Edmund (1970b), « Zur Logik der Zeichen (Semiotik) » (1890), in HUSSERL, Edmund (1970a)

HUSSERL, Edmund (1970c), «<Zum Begriff der Operation> » (1891), in HUSSERL, Edmund (1970a).

HUSSERL, Edmund (1970d), « <Das Imaginäre in der Mathematik> » (1901), in HUSSERL, Edmund (1970a)

HUSSERL, Edmund (1970e), « <Da gebiet eines Axiomensystem - Axiomensystem -

Operationssystem » (1901), in HUSSERL, Edmund (1970a).

HUSSERL, Edmund (1992), Philosophie de l'arithmétique. Recherches psychologiques et logiques, trad. J. English (1972), Paris : PUF.

HUSSERL, Edmund (1995), Articles sur la logique (1891-1910), trad. J. English (1975), Paris : PUF.

ISAAC, Manuel Gustavo (2012), « Saussure ou l'impasse de l'objet », Revue synergie Pays riverains de la mer baltique, 9, 41-52.

LAMBEK, Joachim (1958), « The mathematics of sentence structure », American Mathematical Monthly, 65, 154-170.

LECOMTE, Alain (2008), « La "grammaire pure" : une grammaire des normes », in Jean-Marie Lardic (dir.), La logique et les normes, Hommage à J. L. Gardies, Grenoble : Recherches sur la philosophie et le langage, Université Pierre Mendès-France, 111-137.

LECOMTE, Alain (2011), Meaning, Logic and Ludics, London : Imperial College Press.

RUSSELL, Bertrand \& WHITEHEAD, Alfred North (1910), Principia mathematica, 3 vol., Cambridge: CUP. SAUSSURE, Ferdinand de (1980), Cours de linguistique générale (1916), édition critique préparée par T. de Mauro, Paris : Payot.

SAUSSURE, Ferdinand de (1968), Cours de linguistique générale, édition critique par R. Engler, t. 1, Wiesbaden : Otto Harrassowitz. 
SAUSSURE, Ferdinand de (1974), Cours de linguistique générale, édition critique par R. Engler, t. 2,

Wiesbaden : Otto Harrassowitz.

SAUSSURE, Ferdinand de (2002), Ecrits de linguistique générale, texte établi et édité par S. Bouquet et

R. Engler, Paris : Gallimard.

SAUSSURE, Ferdinand de (2011), Science du langage. De la double essence du langage, édition établie par

R. Amacker, Genève : Droz.

\section{NOTES}

1. Leurs ouvrages programmatiques respectifs sont le Cours de linguistique générale (1916), la Begriffsschrift (1879) et les Logische Untersuchungen (1901) - ouvrages dans lesquels on trouve respectivement formulés les projets de fonder la linguistique générale pour Saussure, l'arithmétique sur la logique pour Frege, ou encore, une nouvelle approche du sens pour Husserl, celle de la phénoménologie.

2. Chez Aristote, il s'agit de la proposition apophantique, laquelle consiste à « affirmer quelque chose de quelque chose, ou [à] nier quelque chose de quelque chose. » (Aristote, $2004: 17 \mathrm{a}, 21$ )

3. Se reporter également au passage suivant de la Métaphysique, E, 4: «(...) le vrai, c'est l'affirmation de la composition réelle du sujet et de l'attribut, et la négation de leur séparation réelle ; le faux, c'est la contradiction de cette affirmation et de cette négation. » (Aristote, 1991 : 1027b, 20-22)

4. Le point est litigieux en raison de la question de l'interprétation via une fonction - dimension absente chez Frege, mais par contre, à la base de la notion de modélisation en théorie des modèles (reste néanmoins qu'il y a chez Frege dichotomisation et dualisation de la syntaxe et de la sémantique). Sur ce point, se reporter à l'article de van Heijenoort (1967).

5. Lorsqu'il conçoit ses grammaires catégorielles, Lambek (1958) affirme s'inscrire dans la continuité du travail de Bar-Hillel (1953) qui, quant à lui se revendique des travaux de la logique polonaise (Ajdukiewicz, 1935), tradition elle-même inspirée des Recherches logiques de Husserl au regard de leur conception du sens (Lecomte, 2008). Quant à la dimension preuve-théorique d'une telle filiation, elle se justifie notamment du fait que les grammaires catégorielles correspondent au fragment non-commutatif de la logique linéaire multiplicative (MNLL). Sur tous ces points, se reporter à Lecomte (2011).

6. On retrouve là toujours la même ambition fondationnaliste. Mais cette fois couplée à une conception forte de ce qu'est un système formel de déduction - à savoir : l'idée de la possibilité d'un système de science axiomatisé, primitif et clos, totalisant par clôture déductive, justement, l'ensemble des «lois logiques possibles en général ». Autrement dit, l'idée de la possibilité d'un réductionnisme nomologique basé uniquement sur la conscience de légalité idéale. Une telle conception est récurrente dans les travaux sur les systèmes d'axiomes (Husserl, 1970a) - travaux contemporains des Recherches logiques.

7. On peut néanmoins déjà souligner à ce stade qu'assigner pour fonction à la logique pure l'élucidation des conditions essentielles a priori de l'adéquation à l'objectité signifiée, et par là, la concevoir comme science des unités de significations idéales exprimant les lois de la pensée pure, engage une hiérarchisation nomologique où l'intensionnalité formelle des catégories prime sur le corrélat intentionnel de la signification. Et c'est en ce sens, certainement, qu'il faut entendre la définition de la logique comme la «science des conditions de possibilité idéales » (Husserl, 1900 ; Husserl 1901d) - cf. 2.1.1.

8. $C f$. le commentaire du traducteur (J. English) : «La Bedeutung est le corrélat objectif d'une visée, d'une Meinung, qui privilégie pour le thématiser un certain aspect des phénomènes, en le 
séparant des autres aspects auxquels il renvoie et dont elle fait implicitement ou délibérément abstraction. » (Husserl, 1992 : 39)

9. Notez qu'à ce titre, les Recherches logiques opèrent une double réarticulation du triangle sémiotique : premièrement, la syntaxe y est absorbée par le pôle (moniste, alors) de la pensée comme calcul du sens ; deuxièmement, cela se produit avec la réintervention parallèle (ou mieux, en simultané) de la question du remplissement, donc du visé référentiel - cf. 2.1.1.

10. Dans sa dimension objective, elle sera pour ainsi dire confondue avec le référent par assimilation sur le plan sémantique (Frege, 1892a). Sur la possibilité des représentations objectives, se reporter à Frege (1884: \$27).

11. L'illusion est, pour Frege, celle de tout idéalisme - au premier rang desquels celui de Husserl (Frege, 1894). Précisément, ce que critique Frege chez Husserl (1891a), c'est le hiatus, ou mieux, l'impossibilité d'instaurer un rapport d'équivalence logique entre représentations propres et symbolisme conceptuel - gage supposé de leur substituabilité réciproque. Selon Frege, il n'y a pas ici de commune mesure. Et en croyant le contraire, on confondrait en fait concept et représentation.

12. Husserl (1893) le formule clairement : « Le signe et ce qui est désigné sont ici [à savoir : dans l'écriture algébrique] totalement étrangers par leurs contenus et ne sont liés que par association. Le signe n'intuitionne donc pas ce qui est pensé, il ne fait que renvoyer.» (Husserl, 1995 : 143) Cela valant même, en termes linguistiques, comme opérateur de démarcation du formel envers le naturel - la fonction des signes dans les langues naturelles (en tant que donnés sensibles) se réduisant, selon Husserl (1891 : 273) à celle d'exprimer les pensées.

13. On constate paradoxalement que c'est donc par suspension de la signification des signes que le symbolisme pur de la syntaxe devient producteur de sens : de par sa concrétude dépourvue de signification, il est aussi sans limites dans son potentiel de... symbolisation : " un seul et même système de la symbolique peut servir à plusieurs systèmes de concepts, qui, différents par leurs contenus, ne présentent des analogies que dans la forme de leur construction. » (Husserl, 1992: 319)

14. L'implication de la dichotomisation de la syntaxe et de la sémantique par l'extensionnalisation de la logique frégéenne procède fondamentalement de deux étapes: premièrement, le développement de la théorie du concept et des dichotomies qui la constituent (propriétés / concepts et objet / concept) ; deuxièmement, le tournant analytique et prédicatif ou fonctionnel opéré sur le plan de la théorie de la proposition. Ces deux étapes font l'objet des Fondements de l'arithmétique et des trois articles des années 1891-1892 (Frege, 1891, 1892a, 1892b) qui composent ainsi le socle de la seconde idéographie (Frege, 1893, 1903).

15. La référence aux éditions critiques d'Engler se fait par renvoi à la page et numération alphabétique de la colonne. Les colonnes b-e sont principalement les notes des auditeurs des Cours I (1907), II (1908-1909), III (1910-1911) ; la colonne f est constituée de notes personnelles de Saussure. On donne à chaque fois, séparée par une barre oblique, la correspondance avec l'édition Payot, ou, le cas échéant, avec l'édition Gallimard. Idem pour la concordance entre les éditions Amacker et Gallimard de l'ensemble de textes coté «Arch. de Saussure 372 " à la Bibliothèque de Genève.

16. «[...] tout est corollaire l'un de l'autre en linguistique statique [...]. La langue est un système serré, et la théorie doit être un système aussi serré que la langue. Là est le point difficile, car ce n'est rien de poser à la suite l'une de l'autre des affirmations, des vues sur la langue ; le tout est de les coordonner en un système. » (Godel, $1957: 29)$ - entretien avec A. Riedlinger, daté du 19 janvier 1909. Je souligne.

17. La référence renvoie aux notes de L. Gautier, prise lors d'un entretien daté du 6 mai 1911 et portant sur le « système de philosophie du langage » de Saussure. Pour en résumer l'articulation : on part d'un ensemble de "vérités fondamentales » (Saussure, 2011: 71 / $2002: 17$ ), ou axiomes, arbitrairement pris pour base du système théorique (Saussure, $2011: 64$ / 2002:34) et on se base 
sur la langue conçue comme "système libre ne dépendant que de rapports logiques "; on applique ensuite à cette langue abstraite (Saussure, 1968 : 173f / $2002: 334$ ) la «saine logique mathématique " (Saussure, 2011:64 / 2002:34) afin d'en exprimer les valeurs, quantités et rapports par des formules mathématiques (Saussure, $1974: 22 \mathrm{~b} / 2002: 206$ ) - Saussure va jusqu'à dire à ce propos : «Il n'y a pas d'expression simple pour les choses à distinguer primairement en linguistique; il ne peut y en avoir. L'expression simple sera algébrique ou ne sera pas.» (Saussure, 1974 : 29b / 2002 : 236) ; enfin, de la «trigonométrie rigoureuse » (Godel, $1957: 141$ ) de ces formules, on déduit les différences sémantiques des éléments du système-langue.

18. «Si un linguiste [...] nous prouvait qu'il existe dans la langue un premier objet tangible, absolument quelconque, mais antérieur à l'analyse et non postérieur à celle-ci, [...] non seulement nous cesserions d'écrire, mais ce []." (Saussure, 1974: 27b / 2002: 227). Voir également Saussure (2011: 83 / $2002: 83)$.

19. «<Ce n'est que dans [biffé]> la mesure où l'objet extérieur est signe < (est perçu comme signe)> qu'il <implique [],> fait partie du langage à titre quelconque. » (Saussure, 1968: 63f / $2002: 213)$

20. C'est le préjugé commun: "Dès qu'il est question quelque part de langue, on voit arriver le mot et le sens (ou le signe et le sens) comme si c'était ce qui résume tout, mais en outre toujours [... ] ce qu'il y a de plus grossier en sémiologie : [...] le cas où il y a un troisième élément incontestable dans l'association psychologique du sème [...].» (Saussure, 1974: 36b-37a / 2002 : 106). Or, selon Saussure: "Si un objet pouvait, où que ce soit, être le terme sur lequel est fixé le signe, la linguistique cesserait immédiatement d'être ce qu'elle est, depuis <le sommet> jusqu'à <la base> [...]. » (Saussure, $1968: 148 \mathrm{f} / 2002: 230$ )

21. Quand le référent intervient comme troisième élément externe à l'unité interne ( $\mathrm{Sa} / \mathrm{Sé}) \mathrm{du}$ signe linguistique auquel il est relié, le signe en question "échappe à la loi générale du signe » (Saussure, 1974: 37a / 2002: 106). Ce cas limite de la sémiologie linguistique, Saussure le dénomme « onymique »-cf. la définition donnée par (Engler, $1968: 37$ ).

22. «Enfin, il est à peine besoin de dire que la différence des termes qui fait le système d'une langue ne correspond nulle part, fût-ce dans la langue la plus parfaite, aux rapports véritables entre les choses; et que par conséquent il n'y a aucune raison d'attendre que les termes s'appliquent complètement ou même très incomplètement à des objets définis, matériels ou autres. » (Saussure, $2011: 203$ / $2002: 76$ )

23. Le "problème de l'intentionnalité" est développée au fragment 107 de l'édition Amacker intitulé par Saussure "Question de synonymie (suite)» (Saussure, 2011 : 200-204), fragment correspondant au paragraphe 26 de l'édition Gallimard (Saussure, $2002: 75-76$ ) - voir également Saussure (2011: 208 / 2002: 38). Quand à l'impossibilité de développer une sémantique conceptuelle sur la base de la sémiologie de Saussure, pour une analyse de détail, on renvoie à Isaac (2012).

24. Les éditeurs $d u$ Cours parlent $d u$ système des valeurs linguistiques comme «système d'équivalence entre choses d'ordre différent " (Saussure, $1980: 115$ ). C'est sur la base de cette équivalence entre concepts et images acoustiques (Saussure, 1968: 253b) que la langue est concevable comme forme (Saussure, 1980 : 157) - et ce, par opposition aux deux masses a-morphes que sont censés être pensée et matière phonique antérieurement à leur "articulation " linguistique (Saussure, 1968: 253b-e / 1980: 156-157), masses non articulées entre elles et inarticulées en elles (Godel, $1957: 213$ ).

25. La conséquence de cette dynamique internalisée de la syntaxe et de la sémantique, c'est la clôture du système-langue. Cette clôture s'opère au niveau de la sémantique du syntagme, par rétroaction du second principe sur le premier, au point d'équilibre du système de la langue point figuré par l'orthogonalité des deux principes (Saussure, 1980: ix), laquelle concrétise le " principe paradoxal » de tout système de valeurs : échanger contre du dissemblable et comparer à du similaire (Saussure, 1980:159) -, la linéarité généralise l'arbitraire sémiologique en 
motivant sémantiquement les composants du syntagme par la stabilisation contextuelle fonction de contraintes compositionnelles - de leurs interactions internes (Saussure, 1968 : 158b, 313be, 314be, 312be ; Godel, $1957: 140,210$ ). Cela signifie que la correspondance signifiant / signifié ne vaut et « n'est complète que pour l'ensemble » syntagmatique (Saussure, 1968:316b). À terme, on a là, au niveau de la sémantique du syntagme, la condition de complétude interne d'un « système qui ne connaît que son ordre propre » (Saussure, $1980: 43$ ) répondant à l'exigence d'exhaustivité formulée par T. de Mauro, à savoir, de «considérer la partie en rapport à cette totalité qui lui donne sa valeur, ou bien en rapport à tout le système linguistique. » (Saussure, $1980: \mathrm{x})$

26. Le système du projet sémiologique aboutit à une contradiction dont découle notamment l'impossibilité de lui coordonner une sémantique vérifiant le principe de compositionnalité. Sur l'établissement de cette impossibilité, se reporter à Isaac (2012). Etablir cette impossibilité suppose à un moment de forcer la sémiologie saussurienne à intégrer une sémantique extensionnelle. Pour cette raison, par contraposition, l'échec du projet sémiologique admet une lecture positive: c'est à une théorie de la signification internalisant l'interaction syntaxe / sémantique sur un mode moniste et intensionnel que doit correspondre le projet sémiologique, ses principes, son système. Avec la Ludique (Girard, 2001 ; 2007) pour paradigme, on a la base d'un nouveau projet. Pour une introduction via la logique linéaire, ses modèles, et les réseaux de preuves pour la logique linéaire multiplicative, se reporter à Curien (2004).

\section{RÉSUMÉS}

Cet article a pour objet l'analyse de trois types de théorisations de la signification basées sur un modèle binaire du signe. Celles de Frege, Husserl et Saussure. Relevant d'un même paradigme, les deux premières sont confrontées en tant que s'y développent deux conceptions opposées de la signification - extensionnelle chez Frege, intensionnelle chez Husserl - contribuant à la mise en place, selon des perspectives opposées, de la dualisation de la syntaxe et de la sémantique. Relativement à cette conséquence, leur paradigme est ensuite opposé à celui de la sémiologie structurale de Saussure dans laquelle l'interaction de la syntaxe et de la sémantique est internalisée au niveau du système qu'intègrent les signes comme éléments. De sorte que ce que l'on vise au terme de cet article, c'est à mettre au jour une explication possible de la raison de la divergence entre deux types de conceptions de la sémantique qui organiseront le développement de courants concurrents tout au long de l'histoire de la linguistique du $20^{\mathrm{e}}$ siècle, l'une formelle et mathématisée, l'autre structurale.

This paper analyses three different theories of meaning based on a binary model of the sign. The ones of Frege, Husserl and Saussure. The two first ones belong to a same paradigm. They develop two opposite conceptions of meaning - extensional Frege's case, intensionnal in Husserl's one which contributes to set up, with opposite perspectives, the dualisation of syntax and semantics. Consequently, their paradigm is then opposed to the structural semiology of Saussure in which the interaction between syntax and semantics is internalised at the level of the system that the signs integrate. The purpose of this paper is therefore to explicit a possible reason for the divergence of two major conceptions of meaning that have organised the development of concurrent trends all along the linguistics of the $20^{\text {th }}$ century, a formal one and a structural one. 
INDEX

Keywords : Theory of sign, theory of meaning, syntax / semantics, intension / extension, semiology, ideography, phenomenology

Mots-clés : Théorie du signe, théorie de la signification, syntaxe / sémantique, intension / extension, sémiologie, idéographie, phénoménologie

\section{AUTEUR}

\section{MANUEL GUSTAVO ISAAC}

Université Denis Diderot - Paris 7

Laboratoire d'histoire des théories linguistiques (UMR CNRS 7597)

mg.isaac@voila.fr 